\title{
STOCHASTIC CONTROLLABILITY OF LINEAR SYSTEMS WITH STATE DELAYS ${ }^{\dagger}$
}

\author{
JERZY KLAMKA \\ Institute of Control Engineering, Silesian University of Technology \\ ul. Akademicka 16, 44-100 Gliwice, Poland \\ e-mail: jerzy.klamka@polsl.pl
}

\begin{abstract}
A class of finite-dimensional stationary dynamic control systems described by linear stochastic ordinary differential state equations with a single point delay in the state variables is considered. Using a theorem and methods adopted directly from deterministic controllability problems, necessary and sufficient conditions for various kinds of stochastic relative controllability are formulated and proved. It will be demonstrated that under suitable assumptions the relative controllability of an associated deterministic linear dynamic system is equivalent to the stochastic relative exact controllability and the stochastic relative approximate controllability of the original linear stochastic dynamic system. Some remarks and comments on the existing results for the controllability of linear dynamic systems with delays are also presented. Finally, a minimum energy control problem for a stochastic dynamic system is formulated and solved.
\end{abstract}

Keywords: controllability, linear control systems, stochastic control systems, delayed state variables, minimum energy control

\section{Introduction}

Controllability is one of the fundamental concepts in mathematical control theory and plays an important role in both deterministic and stochastic control theories (Klamka, 1991; Klamka, 1993; Mahmudov, 2003; Mahmudov and Denker, 2000). Controllability is a qualitative property of dynamic control systems and is of particular importance in control theory. Systematic studies of controllability started at the beginning of the 1960s, when the theory of controllability based on the state space description for both time-invariant and time-varying linear control systems was worked out. Roughly speaking, controllability generally means that it is possible to steer a dynamic control system from an arbitrary initial state to an arbitrary final state using a set of admissible controls. In the literature there are many different definitions of controllability for both linear (Klamka, 1991; Klamka, 1993; Mahmudov, 2001; Mahmudov and Denker, 2000) and nonlinear dynamic systems (Klamka, 2000; Mahmudov, 2002; Mahmudov, 2003; Mahmudov and Zorlu, 2003), which do depend on the class of dynamic control systems and the set of admissible controls

$\dagger$ This work was supported by the Ministry of Science and Higher Education under the grant no. 8T11A 00227.
(Klamka, 1991; Klamka, 1996). Therefore, for linear and nonlinear deterministic dynamic systems there exist many different necessary and sufficient conditions for global and local controllabilities (Klamka, 1991; Klamka, 1993; Klamka, 1996; Klamka, 2000).

In recent years controllability problems for various types of linear dynamic systems have been considered in many publications and monographs. An extensive list of these publications can be found, e.g., in the monograph (Klamka, 1991) or in the survey papers (Klamka, 1993; Klamka, 1996; Klamka, 2000). However, it should be emphasized that most works in this direction are mainly concerned with deterministic controllability problems for finite-dimensional linear dynamic systems with unconstrained controls and without delays

For stochastic control systems (both linear and nonlinear) the situation is by far less satisfactory. In recent years the extensions of deterministic controllability concepts to stochastic control systems have been discussed only in a limited number of publications. In the papers (Bashirov and Kerimov, 1997; Bashirov and Mahmudov, 1999; Ehrhard and Kliemann, 1982; Mahmudov, 2001; Mahmudov and Denker, 2000; Zabczyk, 1991) different kinds of stochastic controllability were discussed for linear finite dimensional stationary and nonstation- 
ary control systems. The papers (Fernandez-Cara et al., 1999; Kim Jong Uhn, 2004; Mahmudov, 2001; Mahmudov, 2003) are devoted to a systematic study of approximate and exact stochastic controllability for linear infinite dimensional control systems defined in Hilbert spaces. Stochastic controllability for finite dimensional nonlinear stochastic systems was discussed in (Arapostathis et al., 2001; Balasubramaniam and Dauer, 2001; Mahmudov and Zorlu, 2003; Sunahara et al., 1974; Sunahara et al., 1975). Using the theory of bounded nonlinear operators and linear semigroups, various different types of stochastic controllability for nonlinear infinite dimensional control systems defined in Hilbert spaces were considered in (Mahmudov, 2002; Mahmudov, 2003). In (Klamka and Socha, 1977; Klamka and Socha, 1980), Lyapunov techniques were used to formulate and prove sufficient conditions for stochastic controllability of nonlinear finite dimensional stochastic systems with point delays in state variables. Moreover, it should be pointed out that the functional analysis approach to stochastic controllability problems is also extensively discussed for both linear and nonlinear stochastic control systems in the papers (Fernandez-Cara et al., 1999; Kim Jong Uhn, 2004; Mahmudov, 2001; Mahmudov, 2002; Mahmudov, 2003; Subramaniam and Balachandran, 2002).

In the present paper we shall study stochastic controllability problems for linear dynamic systems, which are natural generalizations of controllability concepts well known in the theory of infinite dimensional control systems (Klamka, 1991; Klamka, 1993, Ch. 3). Specifically we shall consider stochastic relative exact and approximate controllability problems for finite-dimensional linear stationary dynamic systems with single constant point delays in the state variables described by stochastic ordinary differential state equations. More precisely, using techniques similar to those presented in (Mahmudov, 2001; Mahmudov, 2001; Mahmudov and Denker, 2000), we shall formulate and prove necessary and sufficient conditions for stochastic relative exact controllability in a prescribed time interval for linear stationary stochastic dynamic systems with one constant point delay in the state variables.

Roughly speaking, it will be proved that under suitable assumptions the relative controllability of a deterministic linear associated dynamic system is equivalent to the stochastic relative exact controllability and the stochastic relative approximate controllability of the original linear stochastic dynamic system. This is a generalization of some previous results concerning the stochastic controllability of linear dynamic systems without delays in the control (Mahmudov, 2001; Mahmudov, 2001; Mahmudov and Denker, 2000) to a control delayed case. It is well known (Klamka, 1991) that the controllability concept for linear dynamic systems is strongly connected with the socalled minimum energy control problem. Therefore, us- ing a quite general method presented in (Klamka, 1991) and under the assumption that the stochastic dynamic system is stochastically relatively exactly controllable, a minimum energy control problem is formulated and solved.

The paper is organized as follows: Section 2 contains the mathematical model of a linear, stationary stochastic dynamic system with a single constant point delay in the state variables. Moreover, in this section the basic notation, definitions of stochastic relative exact controllability and stochastic approximate relative controllability as well as some preliminary results are included. In Section 3, using results and methods taken directly from deterministic controllability problems, necessary and sufficient conditions for exact and approximate stochastic relative controllability are formulated and proved. Section 4 is devoted to the study of the minimum energy control problem. In this section we use some optimization methods to solve the so-called minimum energy control problem and to show a relevant analytic formula. Section 5 presents a simple numerical example which illustrates the theoretical deliberations. Finally, Section 6 contains concluding remarks and provides some open controllability problems for more general stochastic dynamic systems.

\section{System Description}

Throughout this paper, unless otherwise specified, we use the following standard notation:. Let $(\Omega, F, P)$ be a complete probability space with a probability measure $P$ on $\Omega$ and a filtration $\left\{F_{t} \mid t \in[0, T]\right\}$ generated by an $n$ dimensional Wiener process $\{w(s): 0 \leq s \leq t\}$ defined on the probability space $(\Omega, F, P)$.

Let $L_{2}\left(\Omega, F_{t}, \mathbb{R}^{n}\right)$ denote the Hilbert space of all $F_{t^{-}}$ measurable square integrable random variables with values in $\mathbb{R}^{n}$. Moreover, let $L_{2}^{F}\left([0, T], \mathbb{R}^{n}\right)$ denote the Hilbert space of all square integrable and $F_{t}$-measurable processes with values in $\mathbb{R}^{n}$. We write $x_{t}=x(t+s)$ for $s \in[-h, 0]$ to denote the segment of the trajectory, i.e., $x_{t} \in L_{2}^{F}\left([-h, 0], L_{2}\left(\Omega, F_{t}, \mathbb{R}^{n}\right)\right)$.

In the theory of linear, finite-dimensional, timeinvariant stochastic dynamic control systems, we use the mathematical model given by the following stochastic ordinary differential state equation with a single point delay in the state variable:

$$
\begin{array}{r}
\mathrm{d} x(t)=\left(A_{0} x(t)+A_{1} x(t-h)+B_{0} u(t)\right) \mathrm{d} t+\sigma \mathrm{d} w(t) \\
\text { for } t \in[0, T], \quad T>h
\end{array}
$$

given the function initial condition

$$
x_{0} \in L_{2}^{F}\left([-h, 0], L_{2}\left(\Omega, F_{T}, \mathbb{R}^{n}\right)\right),
$$

where the state $x(t) \in L_{2}\left(\Omega, F_{t}, \mathbb{R}^{n}\right)=X$ and the values of the control $u(t) \in \mathbb{R}^{m}=U, A_{0}$ and $A_{1}$ are $n \times n$ dimensional constant matrices, $B_{0}$ is a $n \times m$ dimensional constant matrix, $\sigma$ is an $n \times n$ dimensional constant matrix, and $h>0$ is a constant point delay. 
In the sequel, for simplicity, we shall assume that the set of admissible controls is $U_{\mathrm{ad}}=L_{2}^{F}\left([0, T], \mathbb{R}^{m}\right)$.

It is well known (see, e.g., (Mahmudov, 2001; Mahmudov, 2001; Mahmudov and Denker, 2000) or (Mahmudov and Zorlu, 2003) for details) that for a given initial condition (2) and any admissible control $u \in U_{\text {ad }}$ for $t \in[0, T]$ there exists a unique solution $x\left(t ; x_{0}, u\right) \in$ $L_{2}\left(\Omega, F_{t}, \mathbb{R}^{n}\right)$ of the linear stochastic differential state equation (1) which can be represented in every time interval $t \in[k h,(k+1) h), k=0,1,2, \ldots$ by the following integral formula:

$$
\begin{aligned}
x\left(t ; x_{0}, u\right)= & x\left(k h ; x_{0}, u\right) \\
& +\int_{k h}^{t}\left(A_{0} x\left(s ; x_{0}, u\right)+A_{1} x\left(s-h ; x_{0}, u\right)\right) \mathrm{d} s \\
& +\int_{k h}^{t} B_{0} u(s) \mathrm{d} s+\int_{k h}^{t} \sigma \mathrm{d} w(s) .
\end{aligned}
$$

Thus, taking into account the above integral formula and using the well-known method of steps, we obtain the explicit solution of the delayed state equation (1) for $t>0$ in the following compact form (Klamka, 1991, Ch. 4):

$$
\begin{aligned}
x\left(t ; x_{0}, u\right)= & x\left(t ; x_{0}, 0\right)+\int_{0}^{t} F(t-s) B_{0} u(s) \mathrm{d} s \\
& +\int_{0}^{t} F(t-s) \sigma \mathrm{d} w(s),
\end{aligned}
$$

where $F(t)$ is the $n \times n$ dimensional fundamental matrix for the delayed state equation (1), which satisfies the matrix integral equation

$$
F(t)=I+\int_{0}^{t} F(s) A_{0} \mathrm{~d} s+\int_{0}^{t-h} F(s) A_{1} \mathrm{~d} s
$$

for $t>0$, with the initial conditions

$$
F(0)=I, \quad F(t)=0 \text { for } t<0 .
$$

Moreover, for $t>0, x\left(t ; x_{0}, 0\right)$ is given by

$$
\begin{aligned}
x\left(t ; x_{0}, 0\right)= & \exp \left(A_{0} t\right) x_{0}(0) \\
& +\int_{-h}^{0} F(t-s-h) A_{1} x_{0}(s) \mathrm{d} s
\end{aligned}
$$

or, equivalently,

$$
\begin{aligned}
x\left(t ; x_{0}, 0\right)= & \exp \left(A_{0} t\right) x_{0}(0) \\
& \left.+\int_{0}^{h} F(t-s)\right) A_{1} x_{0}(s-h) \mathrm{d} s .
\end{aligned}
$$

Let us denote by $M^{*}$ the transposition of a given arbitrary matrix $M$. Now, for a given final time $T>$ $h$, taking into account the form of the integral solution $x\left(t ; x_{0}, u\right)$, let us introduce the following operators and sets (Klamka, 1991, Ch. 4).

Define the bounded linear control operator $L_{T} \in$ $L\left(L_{2}^{F}\left([0, T], \mathbb{R}^{m}\right), L_{2}\left(\Omega, F_{T}, \mathbb{R}^{n}\right)\right)$ by

$$
\begin{aligned}
L_{T} u= & \int_{0}^{h} \exp \left(A_{0}(T-s)\right) B_{0} u(s) \mathrm{d} s \\
& +\int_{h}^{T} F(T-s) B_{0} u(s) \mathrm{d} s .
\end{aligned}
$$

Its adjoint bounded linear operator $L_{T}^{*} \in$ $L_{2}\left(\Omega, F_{T}, \mathbb{R}^{n}\right) \rightarrow L_{2}^{F}\left([0, T], \mathbb{R}^{m}\right)$ has the following form:

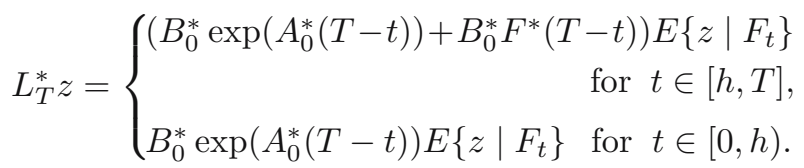

Moreover, we define the set of all the states reachable in the final time $T$ from a given initial state $x_{0} \in$ $L_{2}\left([-h, 0], \mathbb{R}^{n}\right)$, using a set of admissible controls,

$$
\begin{aligned}
& R_{T}\left(U_{\mathrm{ad}}\right)=\left\{x\left(T ; x_{0}, u\right) \in L_{2}\left(\Omega, F_{T}, R^{n}\right): u \in U_{\mathrm{ad}}\right\} \\
& =\left\{\begin{array}{r}
x\left(t ; x_{0}, 0\right)+\operatorname{Im} L_{T}+\int_{0}^{T} \exp \left(A_{0}(T-s)\right) \sigma \mathrm{d} w(s) \\
x\left(t ; x_{0}, 0\right)+\operatorname{Im} L_{T}+\int_{0}^{T} F(T-s) \sigma \mathrm{d} w(s) \\
\text { for } T \leq h,
\end{array}\right.
\end{aligned}
$$

Finally, we introduce the concept of the linear controllability operator (Klamka, 1991; Klamka, 1993; Mahmudov, 2001; Mahmudov and Denker, 2000) $C_{T} \in$ $L\left(L_{2}\left(\Omega, F_{T}, \mathbb{R}^{n}\right), L_{2}\left(\Omega, F_{T}, \mathbb{R}^{n}\right)\right)$, which is closely related to the control operator $L_{T}$ and is defined by

$$
\begin{aligned}
& C_{T}=L_{T} L_{T}^{*} \\
& = \begin{cases}\int_{0}^{T} \exp \left(A_{0}(T-t)\right) B_{0} B_{0}^{*} \exp \left(A_{0}^{*}(T-t)\right) E\left\{\cdot \mid F_{t}\right\} \mathrm{d} t \\
\int_{h}^{T} F(T-t) B_{0} B_{0}^{*} F^{*}(T-t) E\left\{\cdot \mid F_{t}\right\} \mathrm{d} t \\
+\int_{0}^{h} \exp \left(A_{0}(T-t)\right) B_{0} B_{0}^{*} & \text { for } T \leq h, \\
\quad \times \exp \left(A_{0}^{*}(T-t)\right) E\left\{\cdot \mid F_{t}\right\} \mathrm{d} t & \text { for } T>h .\end{cases}
\end{aligned}
$$


Moreover, let us recall the $n \times n$-dimensional relative controllability matrix (Klamka, 1991, Ch. 4),

$$
G_{T}=\left\{\begin{array}{lr}
\int_{0}^{T} \exp \left(A_{0}(T-t)\right) B_{0} B_{0}^{*} \exp \left(A_{0}^{*}(T-t)\right) \mathrm{d} t \\
\int_{h}^{T} F(T-t) B_{0} B_{0}^{*} F^{*}(T-t) \mathrm{d} t & \text { for } T \leq h, \\
+\int_{0}^{h} \exp \left(A_{0}(T-t)\right) B_{0} B_{0}^{*} \exp \left(A_{0}^{*}(T-t)\right) \mathrm{d} t & \text { for } T>h .
\end{array}\right.
$$

In the proofs of the main results we shall also use the relative controllability operator $C_{T}(s)$ and the relative controllability matrix $G_{T}(s)$, both depending on time $s \in$ $[0, T]$. The former is defined as

$$
\begin{aligned}
& C_{T}(s)=L_{T}(s) L_{T}^{*}(s) \\
& = \begin{cases}\int_{s}^{T} \exp \left(A_{0}(T-t)\right) B_{0} B_{0}^{*} \exp \left(A_{0}^{*}(T-t)\right) E\left\{\cdot \mid F_{t}\right\} \mathrm{d} t \\
\int_{s}^{T} F(T-t) B_{0} B_{0}^{*} F^{*}(T-t) E\left\{\cdot \mid F_{t}\right\} \mathrm{d} t & \text { for } T \leq h, \\
& \int_{0}^{s} \exp \left(A_{0}(T-t)\right) B_{0} B_{0}^{*} \\
& \times \exp \left(A_{0}^{*}(T-t)\right) E\left\{\cdot \mid F_{t}\right\} \mathrm{d} t \text { for } T>h .\end{cases}
\end{aligned}
$$

In turn, the latter is

$$
\begin{aligned}
& G_{T} \begin{array}{lr}
\int_{s}^{T} \exp \left(A_{0}(T-t)\right) B_{0} B_{0}^{*} \exp \left(A_{0}^{*}(T-t)\right) \mathrm{d} t \\
\int_{s}^{T} F(T-t) B_{0} B_{0}^{*} F^{*}(T-t) \mathrm{d} t & \text { for } T \leq h, \\
+\int_{0}^{s} \exp \left(A_{0}(T-t)\right) B_{0} B_{0}^{*} \exp \left(A_{0}^{*}(T-t)\right) \mathrm{d} t
\end{array} \\
& \text { for } T>h .
\end{aligned}
$$

In the theory of dynamic systems with delays in the control or state variables, it is necessary to distinguish between two fundamental concepts of controllability, namely, the relative controllability and the absolute controllability (see, e.g., (Klamka, 1991; Klamka, 1993; Klamka, 2000) for more details). In this paper we shall concentrate on the weaker concept of relative controllability. On the other hand, since for the stochastic dynamic system (1) the state space $L_{2}\left(\Omega, F_{t}, \mathbb{R}^{n}\right)$ is in fact an infinite-dimensional space, we distinguish exact (or strong) controllability and approximate (or weak) controllability. Using the above notation for the stochastic dynamic system (1) we define the following stochastic relative exact and approximate controllability concepts:

Definition 1. The stochastic dynamic system (1) is said to be relatively exactly controllable on $[0, T]$ if

$$
R_{T}\left(U_{\mathrm{ad}}\right)=L_{2}\left(\Omega, F_{T}, \mathbb{R}^{n}\right),
$$

that is, if all the points in $L_{2}\left(\Omega, F_{T}, \mathbb{R}^{n}\right)$ can be exactly reached at time $T$ from any arbitrary initial condition $x_{0} \in$ $L_{2}^{F}\left([-h, 0], L_{2}\left(\Omega, F_{T}, \mathbb{R}^{n}\right)\right)$.

Definition 2. The stochastic dynamic system (1) is said to be relatively approximately controllable on $[0, T]$ if

$$
\overline{R_{T}\left(U_{\mathrm{ad}}\right)}=L_{2}\left(\Omega, F_{T}, \mathbb{R}^{n}\right),
$$

that is, if all the points in $L_{2}\left(\Omega, F_{T}, \mathbb{R}^{n}\right)$ can be approximately reached at time $T$ from any arbitrary initial condition $x_{0} \in L_{2}^{F}\left([-h, 0], L_{2}\left(\Omega, F_{T}, R^{n}\right)\right)$.

Remark 1. From Definitions 1 and 2 it directly follows that the exact relative controllability is generally a stronger concept than the approximate relative controllability. However, it should be mentioned that there are many cases when these two concepts coincide.

Remark 2. Since the stochastic dynamic system (1) is linear, without loss of generality in the above two definitions it is enough to assume the zero initial condition.

Remark 3. It should be pointed out that in the case of delayed states or controls the above controllability concepts depend on the time interval $[0, T]$.

Remark 4. Since for $T \leq 0$ the stochastic dynamic system (1) is in fact a dynamic system without delay, we shall generally assume that $T>h$.

Remark 5. Since the stochastic dynamic system (1) is stationary the controllability matrix $G_{T}(s)$ has the same rank at least for all $s \in[0, T-h]$, cf. (Klamka, 1991, Ch. 4).

Remark 6. From the form of the controllability operator $C_{T}$ it follows immediately that this operator is selfadjoint.

In the sequel we study the relationship between the controllability concepts for the stochastic delayed dynamic system (1) and the controllability of the associated deterministic delayed dynamic system of the following form:

$$
y^{\prime}(t)=A_{0} y(t)+A_{1} y(t-h)+B_{0} v(t) \text { for } t \in[0, T],
$$

where the admissible control $v \in L_{2}\left([0, T], \mathbb{R}^{m}\right)$. 
First of all, following (Klamka, 1991, Ch. 4), we shall introduce the concept of the so-called "defining equation" for the deterministic delayed system (3). Let

$$
Q_{k}(t)=A_{0} Q_{k-1}(t)+A_{1} Q_{k-1}(t-h)
$$

for $k=1,2,3, \ldots$ and $t>0$, with the initial condition

$$
Q_{0}(0)=B_{0}, \quad Q_{0}(t)=0 \text { for } t \neq 0 .
$$

Thus, for example, the sequence of the $n \times n$ dimensional matrices $Q_{k}(t)$ derived from the determining equation has the following form:

$$
\begin{aligned}
& Q_{0}(0)=B_{0}, \quad Q_{0}(j h)=0, \text { for } j=1,2,3, \ldots, \\
& Q_{1}(0)=A_{0} B_{0}, \quad Q_{1}(h)=A_{1} B_{0}, \quad Q_{1}(j h)=0, \\
& \text { for } j=2,3,4, \ldots \\
& Q_{2}(0)=A_{0}^{2} B_{0}, \quad Q_{2}(h)=\left(A_{0} A_{1}+A_{1} A_{0}\right) B_{0}, \\
& Q_{2}(2 h)=A_{1}^{2} B_{0}, \quad Q_{2}(j h)=0, \text { for } j=2,3,4, \ldots \text {. }
\end{aligned}
$$

For notational simplicity,write

$$
\begin{aligned}
& Q_{n}(t ; T) \\
& =\left\{Q_{0}(t), Q 1(t), Q_{2}(t), \ldots, Q_{n-1}(t), \text { for } t \in[0, T)\right\} .
\end{aligned}
$$

Recall the following lemma concerning the relative controllability of the deterministic delayed system (3) in the time interval $[0, T]$ :

Lemma 1. (Klamka, 1991, Ch. 4). The following conditions are equivalent:

(i) the deterministic system (3) is relatively controllable on $[0, T]$,

(ii) the controllability matrix $G_{T}$ is nonsingular,

(iii) $\operatorname{rank} Q_{n}(t ; T)=n$.

Remark 7. It should be pointed out that for linear, timeinvariant dynamic systems without delays the length of the time interval $[0, T]$ is inessential in controllability investigations. However, for linear time-invariant dynamic systems with delays the situation is quite different. The length of the time interval $[0, T]$ acquires an important role. For example, for $T<h$, from the defining equation we have

$$
Q_{n}(t ; T)=\left\{B_{0}, A_{0} B_{0}, A_{0}^{2} B_{0}, \ldots, A_{0}^{n-1} B_{0}\right\} .
$$

Hence, by Lemma 1, the relative controllability of a system with a delay is equivalent to that of a dynamic system without delays.

Now, let us formulate the following auxiliary wellknown lemma, which will be used in the sequel in the proofs of the main results.
Lemma 2. (Mahmudov, 2001; Mahmudov and Denker, 2000; Mahmudov and Zorlu, 2003) For every $z \in$ $L_{2}\left(\Omega, F_{T}, \mathbb{R}^{n}\right)$, there exists a process $q \in L_{2}^{F}([0, T]$, $\left.\mathbb{R}^{n \times n}\right)$ such that

$$
C_{T} z=G_{T} E z+\int_{0}^{T} G_{T}(s) q(s) \mathrm{d} w(s) .
$$

Taking into account the above notation, definitions and lemmas, in the next section we shall formulate and prove the conditions for relative exact and relative approximate controllabilities for the stochastic dynamic system (1).

\section{Stochastic Relative Controllability}

In this section, using the lemmas given in Section 2, we shall formulate and prove the main result of the paper, which says that the stochastic relative exact controllability and, in consequence, also the relative approximate controllability of the stochastic system (1) are in fact equivalent to the relative controllability of the associated linear deterministic system (3).

\section{Theorem 1. The following conditions are equivalent:}

(i) The deterministic system (3) is relatively controllable on $[0, T]$,

(ii) The stochastic system (1) is relatively exactly controllable on $[0, T]$

(iii) The stochastic system (1) is relatively approximately controllable on $[0, T]$.

Proof. (i) $\Rightarrow$ (ii) Let us assume that the deterministic system (3) is relatively controllable on $[0, T]$. Then it is well known (see, e.g., (Klamka, 1991; Klamka, 1993) or (Klamka and Socha, 1977)) that the relative controllability matrix $G_{T}(s)$ is invertible and strictly positive definite at least for all $s \in[0, T-h]$ (Klamka, 1991, Ch. 4). Hence, for some $\gamma>0$ we have

$$
\left\langle G_{T}(s) x, x\right\rangle \geq \gamma\|x\|^{2}
$$

for all $s \in[0, T-h]$ and for all $x \in \mathbb{R}^{n}$. To prove the relative exact controllability of the stochastic system (1) on $[0, T]$, we use the relationship between the the controllability operator $C_{T}$ and the controllability matrix $G_{T}$ given in Lemma 2 to write $E\left\langle C_{T} z, z\right\rangle$ in terms of $\left\langle G_{T} E z, E z\right\rangle$.

First of all, we obtain

$$
\begin{gathered}
E\left\langle C_{T} z, z\right\rangle=E\left\langle G_{T} E z+\int_{0}^{T} G_{T}(s) q(s) \mathrm{d} w(s),\right. \\
\left.E z+\int_{0}^{T} q(s) \mathrm{d} w(s)\right\rangle
\end{gathered}
$$




$$
\begin{aligned}
& =\left\langle G_{T} E z, E z\right\rangle+E \int_{0}^{T}\left\langle G_{T}(s) q(s), q(s)\right\rangle \mathrm{d} s \\
& \geq \gamma\left(\|E z\|^{2}+E \int_{0}^{T}\|q(s)\|^{2} \mathrm{~d} s\right) \\
& =\gamma E\|z\|^{2} .
\end{aligned}
$$

Hence, in the operator sense, we have $C_{T} \geq \gamma I$, which means that the relative controllability operator $C_{T}$ is strictly positive definite and, consequently, that the inverse linear operator $C_{T}^{-1}$ is bounded. Therefore, the stochastic relative exact controllability of the stochastic dynamic system (1) on $[0, T]$ directly follows from the results given in (Klamka, 1991, Ch. 3). Moreover, in the next section, using the fact that the operator $C_{T}^{-1}$ is bounded, we shall construct a control $u^{0}(t), t \in[0, T]$ which steers the stochastic dynamic system (1) from a given initial state $x_{0}$ to a desired final state $x_{T}$ at time $T$.

(ii) $\Rightarrow$ (iii) This implication is obvious (see, e.g., (Klamka, 1991; Mahmudov, 2001; Mahmudov, 2002; Mahmudov, 2003, Ch. 3)).

(iii) $\Rightarrow$ (i) Assume that the stochastic dynamic system (1) is stochastically relatively approximately controllable on $[0, T]$, and hence its controllability operator is positive definite, i.e., $C_{T}>0$ (Klamka, 1991, Ch. 3). Then, using the resolvent operator $R\left(\lambda, C_{T}\right)$ and following directly the functional analysis method given in (Mahmudov, 2001; Mahmudov and Denker, 2000; Mahmudov and Zorlu, 2003) for stochastic dynamic systems without delays, we obtain that the deterministic system (3) is approximately relatively controllable on $[0 . T]$. However, taking into account that the state space for the deterministic dynamic system (3) is finite dimensional, i.e., exact and approximate controllabilities coincide (Klamka, 1991, Ch. 3), we conclude that the deterministic dynamic system (3) is relatively controllable on $[0 . T]$.

Remark 8. Let us observe that for a special case when $T \leq h$, the stochastic relative exact or relative approximate controllability problems in $[0, T]$ for a stochastic dynamic system with a delay in the state variables (1) are reduced to the standard stochastic exact or approximate controllability problems for a stochastic dynamic system without delays in the control (Klamka, 1991, Ch. 4).

Corollary 1. (Mahmudov, 2001; Mahmudov and Denker, 2000) Suppose that $T \leq h$. Then the stochastic dynamic control system (1) is stochastically relatively exactly controllable in $[0, T]$ if and only if

$$
\operatorname{rank}\left[B_{0}, A B_{0}, A^{2} B_{0}, \ldots, A^{n-1} B_{0}\right]=n .
$$

Corollary 2. (Mahmudov and Denker, 2000). A stochastic dynamic system without delay $\left(A_{1}=0\right)$ is stochastically exactly controllable in any time interval if and only if the associated deterministic dynamic system without delay is controllable.

\section{Minimum Energy Control}

The minimum energy control problem is strongly connected with the controllability concept (see, e.g., (Klamka, 1991) for more details). First of all, observe that for an exactly controllable linear control system on $[0, T]$ there exist in general many different admissible controls $u(t)$, defined for $t \in[0, T]$ and transferring the initial state $x_{0}$ to the desired final state $x_{T}$ at a given time $T$. Therefore, we may ask which of these possible admissible controls is an optimal one according to a given criterion. In the sequel, we shall consider the minimum energy control problem for the stochastic dynamic system (1) with the optimality criterion representing the energy of control. This optimality criterion has the following form:

$$
J(u)=E \int_{0}^{T}\|u(t)\|^{2} \mathrm{~d} t .
$$

Theorem 2. Assume that the stochastic dynamic system (1) is relatively exactly controllable on $[0, T]$. Then, for an arbitrary final state $x_{T} \in L_{2}\left(\Omega, F_{T}, \mathbb{R}^{n}\right)$ and an arbitrary matrix $\sigma$, the admissible control

$$
\begin{aligned}
u^{0}(t)=B_{0}^{*} F^{*}(T-t) & E\left\{C _ { T } ^ { - 1 } \left(x_{T}-x\left(T ; x_{0}, 0\right)\right.\right. \\
& \left.\left.-\int_{0}^{T} F(T-s) \sigma \mathrm{d} w(s)\right) \mid F_{t}\right\}
\end{aligned}
$$

defined for $t \in[0, T]$ transfers the delayed dynamic system (1) from a given initial state $x_{0} \in L_{2}\left([-h, 0], \mathbb{R}^{n}\right)$ to the final state $x_{T}$ at time $T$.

Moreover, among all admissible controls $u^{a}(t)$ transferring the initial state $x_{0}$ to the final state $x_{T}$ at time $T$, the control $u^{0}(t)$ minimizes the integral performance index

$$
J(u)=E \int_{0}^{T}\|u(t)\|^{2} \mathrm{~d} t .
$$

Proof. First of all, observe that, since the stochastic dynamic system (1) is relatively exactly controllable on $[0, T]$, the controllability operator $C_{T}$ is invertible and its inverse $C_{T}^{-1}$ is a bounded linear operator, i.e., $C_{T}^{-1} \in$ $L\left(L_{2}\left(\Omega, F_{T}, \mathbb{R}^{n}\right), L_{2}\left(\Omega, F_{T}, \mathbb{R}^{n}\right)\right)$. Moreover,

$$
\begin{aligned}
x\left(t ; x_{0}, u\right)= & x\left(t ; x_{0}, 0\right)+\int_{0}^{t} F(t-s) B_{0} u(s) \mathrm{d} s \\
& +\int_{0}^{t} F(t-s) \sigma \mathrm{d} w(s) .
\end{aligned}
$$


Substituting the control $u^{0}(t), t \in[0, T]$ into the general integral formula for the solution, one can easily obtain

$$
\begin{aligned}
& x\left(t ; x_{0}, u^{0}\right)=x\left(t ; x_{0}, 0\right) \\
& +\int_{0}^{t} F(t-s) B_{0} B_{0}^{*} F^{*}(t-s) E\left\{C_{T}^{-1}\right. \\
& \left.\quad \times\left(x_{T}-x\left(T ; x_{0}, 0\right)-\int_{0}^{T} F(T-s) \sigma \mathrm{d} w(s)\right)\right\} \mid F_{s} \mathrm{~d} s \\
& +\int_{0}^{t} F(t-s) \sigma \mathrm{d} w(s) .
\end{aligned}
$$

Hence, for a given final time $t=T$, we simply have the following equality:

$$
\begin{aligned}
& x\left(T ; x_{0}, u^{0}\right)=x\left(T ; x_{0}, 0\right) \\
& +\int_{0}^{T} F(T-s) B_{0} B_{0}^{*} F^{*}(t-s) E\left\{C_{T}^{-1}\right. \\
& \left.\quad \times\left(x_{T}-x\left(T ; x_{0}, 0\right)-\int_{0}^{T} F(T-s) \sigma \mathrm{d} w(s)\right)\right\} \mid F_{s} \mathrm{~d} s \\
& \quad+\int_{0}^{T} F(T-s) \sigma \mathrm{d} w(s) .
\end{aligned}
$$

Thus, taking into account the form of the operator $C_{T}$, we have

$$
\begin{aligned}
& x\left(T ; x_{0}, u^{0}\right)=x\left(T ; x_{0}, 0\right) \\
& \quad+C_{T} C_{T}^{-1}\left(x_{T}-x\left(T ; x_{0}, 0\right)-\int_{0}^{T} F(T-s) \sigma \mathrm{d} w(s)\right) \\
& \quad+\int_{0}^{T} \exp (A(T-s)) \sigma \mathrm{d} w(s) \\
& =x\left(T ; x_{0}, 0\right)+x_{T}-x\left(T ; x_{0}, 0\right) \\
& \quad-\int_{0}^{T} F(T-s) \sigma \mathrm{d} w(s)+\int_{0}^{T} F(T-s) \sigma \mathrm{d} w(s) \\
& =x_{T} .
\end{aligned}
$$

Therefore, for $t=T$ we see that the control $u^{0}(t)$ transfers the system from the initial state $x_{0} \in L_{2}\left(\Omega, F_{T}, \mathbb{R}^{n}\right)$ to the final state $x_{T} \in L_{2}\left(\Omega, F_{T}, \mathbb{R}^{n}\right)$ at time $T$.

In the second part of the proof, using a general method presented in (Klamka, 1991, Ch. 1), we shall show that the control $u^{0}(t), t \in[0, T]$ is optimal for the performance index $J$. To this end, suppose that $u^{\prime}(t), t \in[0, T]$ is any other admissible control which also steers the initial state $x_{0}$ to the final state $x_{T}$ at time $T$. Hence, since the delayed dynamic system (1) is relatively exactly controllable on $[0, T]$, using the relative controllability operator defined in Section 2, we have

$$
L_{T}\left(u^{0}(\cdot)\right)=L_{T}\left(u^{\prime}(\cdot)\right) .
$$

Using the basic properties of the scalar product in $\mathbb{R}^{n}$ and the form of the relative controllability operator $L_{T}$, we obtain

$$
E \int_{0}^{T}\left\langle\left(u^{\prime}(t)-u^{0}(t)\right), u^{0}(t)\right\rangle \mathrm{d} t=0 .
$$

Moreover, using once again the basic properties of the scalar product in $\mathbb{R}^{n}$, we have

$$
\begin{aligned}
& E \int_{0}^{T}\left\|u^{\prime}(t)\right\|^{2} \mathrm{~d} t \\
& \quad=E \int_{0}^{T}\left\|u^{\prime}(t)-u^{0}(t)\right\|^{2} \mathrm{~d} t+E \int_{0}^{T}\left\|u^{0}(t)\right\|^{2} \mathrm{~d} t .
\end{aligned}
$$

Since

$$
E \int_{0}^{T}\left\|u^{\prime}(t)-u^{0}(t)\right\|^{2} \mathrm{~d} t \geq 0
$$

we conclude that, for any admissible control $u^{\prime}(t), t \in$ $[0, T]$,

$$
E \int_{0}^{T}\left\|u^{0}(t)\right\|^{2} \mathrm{~d} t \leq E \int_{0}^{T}\left\|u^{\prime}(t)\right\|^{2} \mathrm{~d} t .
$$

Hence the control $u^{0}(t), t \in[0, T]$ is optimal control for the performance index $J$, and thus it is a minimum energy control.

\section{Example}

As a simple illustrative example, consider a stochastic delayed dynamic control system of the form (1) defined in a given time interval $[0, T], T>1$, with one constant point delay $h=1$, with an arbitrary $3 \times 3$ dimensional matrix $\sigma$, and with the following constant matrices:

$$
\begin{gathered}
A_{0}=\left[\begin{array}{lll}
1 & 0 & 0 \\
0 & 1 & 0 \\
0 & 0 & 1
\end{array}\right], \quad A_{1}=\left[\begin{array}{ccc}
-1 & 1 & 0 \\
1 & 0 & 1 \\
0 & 1 & 1
\end{array}\right], \\
B_{0}=\left[\begin{array}{ll}
0 & 0 \\
1 & 0 \\
0 & 1
\end{array}\right] .
\end{gathered}
$$


Hence, $n=3, m=2$ and

$$
Q_{3}(t ; T)=\left\{Q_{0}(t), Q_{1}(t), Q_{2}(t), \quad \text { for } t \in[0, T)\right\} .
$$

Moreover, using the notation given in Section 2, we have

$$
\begin{aligned}
& Q_{3}(t ; T) \\
& =\left[Q_{0}(0): Q_{1}(0): Q_{1}(h): Q_{2}(0): Q_{2}(h): Q_{2}(2 h)\right] \\
& =\left[\begin{array}{l:l:}
B_{0} & A_{0} B_{0}
\end{array} A_{1} B_{0}: A_{0}^{2} B_{0}:\left(A_{0} A_{1}+A_{1} A_{0}\right) B_{0}: A_{1}^{2} B_{0}\right] .
\end{aligned}
$$

Substituting the matrices $A_{0}, A_{1}$, and $B_{0}$ given above, we easily obtain

$$
\begin{aligned}
& \operatorname{rank} Q_{3}(t ; T) \\
& \quad=\operatorname{rank}\left[\begin{array}{cc:cc:cc:cc:cc:cc}
0 & 0 & 0 & 0 & 1 & 0 & 0 & 0 & 2 & 0 & -1 & 1 \\
1 & 0 & 1 & 0 & 0 & 1 & 1 & 0 & 0 & 2 & 0 & 1 \\
0 & 1 & 0 & 1 & 1 & 1 & 0 & 1 & 2 & 4 & 2 & 1
\end{array}\right] \\
& \quad=3=n .
\end{aligned}
$$

Hence, by Lemma 1, the deterministic system with delay is relatively controllable in each time interval $[0, T]$ for $T>1$. Therefore, by Theorem 1 , the stochastic dynamic system with delay is stochastically relatively exactly controllable in each time interval $[0, T]$ for $T>1$.

However, since

$$
\begin{aligned}
& \operatorname{rank}\left[\begin{array}{l:l:l:l}
B_{0} & A_{0} B_{0} & A_{0}^{2} B_{0}
\end{array}\right] \\
& \quad=\operatorname{rank}\left[\begin{array}{ll:ll:ll}
0 & 0 & 0 & 0 & 0 & 0 \\
1 & 0 & 1 & 0 & 1 & 0 \\
0 & 1 & 0 & 1 & 0 & 1
\end{array}\right]=2<3=n,
\end{aligned}
$$

the deterministic system without delay is not controllable in any time interval and thus the deterministic system with delay is not relatively controllable in $[0, T]$ for $T \leq 1$. Therefore, by Corollary 1 the stochastic delayed system is not stochastically relatively exactly controllable in each time interval $[0, T]$ for $T \leq 1$.

\section{Concluding Remarks}

In the paper, sufficient conditions for the stochastic relative exact controllability of a linear stationary finitedimensional stochastic dynamic control system with a single constant point delay in the control have been formulated and proved. It should be pointed out that these conditions extend the stochastic exact controllability conditions for dynamic control systems without delays recently published in the papers (Mahmudov, 2001; Mahmudov, 2002; Mahmudov and Denker, 2000) to the case of a constant point delay in the state variables. Finally, it should be pointed out that, using standard techniques presented, e.g., in the monograph (Klamka, 1991, Ch. 4), it is possible to extend the results presented in this paper to nonstationary linear stochastic control systems with many time variable point delays in the state variables or in the control. Extensions to stochastic absolute exact and approximate controllabilities are also possible.

\section{References}

Arapostathis A., George R.K., Ghosh M.K. (2001): On the controllability of a class of nonlinear stochastic systems. Syst. Contr. Lett., Vol. 44, No. 1, pp. 25-34.

Balasubramaniam P. and Dauer J.P. (2001): Controllability of semilinear stochastic evolution equations in Hilbert spaces. - J. Appl. Math. Stoch. Anal., Vol. 14, No. 4 pp. 329-339.

Bashirov A.E., and Kerimov K.R. (1997): On controllability conception for stochastic systems. — SIAM J. Contr. Optim., Vol. 35, No. 2, pp. 348-398.

Bashirov A.E. and Mahmudov N.I. (1999): On concepts of controllability for deterministic and stochastic systems. SIAM J. Contr. Optim., Vol. 37, No. 6, pp. 1808-1821.

Ehrhard M. and Kliemann W. (1982): Controllability of stochastic linear systems. - Syst. Contr. Lett., Vol. 2, No. 2, pp. 145-153.

Fernandez-Cara E., Garrido-Atienza M.J. and Real J. (1999): On the approximate controllability of a stochastic parabolic equation with multiplicative noise. - C.R. Acad. Sci. Paris, t. 328, Sèrie 1, pp. 675-680.

Kim Jong Uhn (2004): Approximate controllability of a stochastic wave equation. - Appl. Math. Optim., Vol. 49, No. 1, pp. 81-98.

Klamka J. (1991): Controllability of Dynamical Systems. Dordrecht: Kluwer Academic.

Klamka J. (1993): Controllability of dynamical systems -A survey. - Arch. Contr. Sci., Vol. 2, No. 3/4, pp. 281-307.

Klamka J. (1996): Constrained controllability of nonlinear systems. - J. Math. Anal. Applic., Vol. 201, No. 2, pp. 365 374.

Klamka J. (2000): Schauder's fixed point theorem in nonlinear controllability problems. - Contr. Cybern., Vol. 29, No. 3 , pp. 377-393.

Klamka J. and Socha L. (1977): Some remarks about stochastic controllability. - IEEE Trans. Automat. Contr., Vol. AC22, No. 5, pp. 880-881.

Klamka J. and Socha L. (1980): Some remarks about stochastic controllability for delayed linear systems. - Int. J. Contr., Vol. 32, No. 3, pp. 561-566.

Mahmudov N.I. (2001): Controllability of linear stochastic systems. - IEEE Trans. Automat. Contr., Vol. AC-46, No. 4, pp. 724-731.

Mahmudov N.I. (2001): Controllability of linear stochastic systems in Hilbert spaces. - J. Math. Anal. Applic., Vol. 259, No. 1, pp. 64-82. 
Mahmudov N.I. (2002): On controllability of semilinear stochastic systems in Hilbert spaces. - IMA J. Mathemat. Contr. Inf., Vol. 19, No. 2, pp. 363-376.

Mahmudov N.I. (2003): Controllability and observability of linear stochastic systems in Hilbert spaces. - Progress in Probability, Vol. 53, No. 1, pp. 151-167.

Mahmudov N.I. (2003): Approximate controllability of semilinear deterministic and stochastic evolution equations in abstract spaces. - SIAM J. Contr. Optim. Vol. 42, No. 5, pp. 1604-1622.

Mahmudov N.I. and Denker A. (2000): On controllability of linear stochastic systems. - Int. J. Contr., Vol. 73, No. 2, pp. 144-151.

Mahmudov N.I. and Zorlu S. (2003): Controllability of nonlinear stochastic systems. - Int. J. Contr., Vol. 76, No. 2, pp. 95-104.
Subramaniam R. and Balachandran K. (2002): Controllability of stochastic Volterra integrodifferential systems. - Korean J. Comput. Appl. Math., Vol. 9, No. 2, pp. 583-589.

Sunahara Y., Kabeuchi T., Asada S., Aihara S. and Kishino K. (1974): On stochastic controllability for nonlinear systems. - IEEE Trans. Automat. Contr., Vol. AC-19, No. 1, pp. 49-54.

Sunahara Y., Aihara S. and Kishino K. (1975): On the stochastic observability and controllability for nonlinear systems. Int. J. Contr., Vol. 22, No. 1, pp. 65-82.

Zabczyk J. (1991): Controllability of stochastic linear systems. — Syst. Contr. Lett., Vol. 1, No. 1, pp. 25-31.

Received: 15 November 2006 Revised: 8 January 2007 